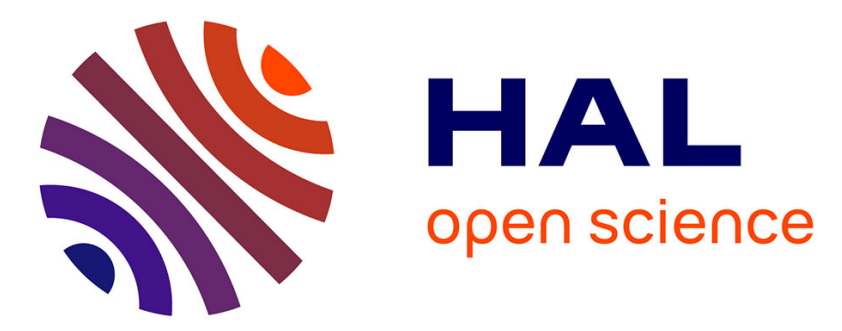

\title{
Colonization and establishment of the rumen microbiota - opportunities to influence productivity and methane emissions
}

\author{
Diego Morgavi, Milka Popova, David Yáñez-Ruiz, Evelyne Forano
}

\section{To cite this version:}

Diego Morgavi, Milka Popova, David Yáñez-Ruiz, Evelyne Forano. Colonization and establishment of the rumen microbiota - opportunities to influence productivity and methane emissions. Improving rumen function, pp.1-26, 2020, 10.19103/AS.2020.0067.02 . hal-02891638

\section{HAL Id: hal-02891638 \\ https://hal.inrae.fr/hal-02891638}

Submitted on 24 Mar 2021

HAL is a multi-disciplinary open access archive for the deposit and dissemination of scientific research documents, whether they are published or not. The documents may come from teaching and research institutions in France or abroad, or from public or private research centers.
L'archive ouverte pluridisciplinaire HAL, est destinée au dépôt et à la diffusion de documents scientifiques de niveau recherche, publiés ou non, émanant des établissements d'enseignement et de recherche français ou étrangers, des laboratoires publics ou privés. 


\section{Colonisation and establishment of the rumen microbiota - opportunities to influence productivity and methane emissions}

Diego P. Morgavi, INRA, France; Milka Popova, INRA, France; David Yañez-Ruiz, CSIC, Spain; and Evelyne Forano, INRA, France

1. Introduction

2. Establishment of the rumen microbiota

Colonisation: from birth (preruminant) to a fully functional rumen Interaction host-microbiota

Weaning and stabilization of the rumen microbiota

Microbial activities and functions

Modes of transmission

Impact of management practices

3. Modulating the gastrointestinal microbiota in young ruminants for health and production

4. Early-life strategies for improving health and production (case studies)

5. Early-life strategies for reducing enteric methane emissions (case studies)

6. Summary and outstanding questions/future trends

7. Where to look for further information

8. References

\section{Introduction}

The gastrointestinal (GIT) microbiota of ruminants cannot be dissociated from the host animal. Gut symbiotic microbes have a critical role in the interaction of the host animal with the surrounding environment, providing fundamental nutritional, immunological and protection services. As for other essential body 'organ,' the GIT microbiota undergoes a series of development stages from early stages of formation until maturity. Differently from the embryogenesis process; however, the 
development of the GIT microbiota starts in earnest at birth and it is characterized by a succession of dynamic communities in the early stages of life (Savage, 1977, Jami et al., 2013, Rey et al., 2014). This process of acquisition of various microbial populations and their evolution within the ecosystem is essential for the correct functioning and interaction of the microbiota with the host (Costello et al., 2012).

Determinism is a strong driver dictating the microbial community structure of the GIT of animals as there is a strong selection by the diet, anatomy and gut physico-chemical conditions (Ley et al., 2008). Yet, stochastic and historical events also influence the assemblage of the GIT microbiota that may have lasting effects in ruminants (Yáñez-Ruiz et al., 2010, Morgavi et al., 2015, Moraïs and Mizrahi, 2019). Here, we review current information in the establishment of the microbiota in the rumen and posterior intestinal tract in young ruminants and its modulation for promoting health and favouring desirable phenotypes.

\section{Establishment of the rumen microbiota}

As the composition of the rumen microbiota directly influences the digestive and metabolic performance of the host animal, many studies have explored the microbial colonization of the rumen from birth to adulthood. These include early work using cultural methods (Fonty et al., 1983, Fonty et al., 1988) to more recent studies using high throughput sequencing methods in calves, lambs and goats (Jami et al., 2013, Rey et al., 2014, Guzman et al., 2015, Wang et al., 2017b, Abecia et al., 2018, Dias et al., 2018). The developing rumen in the newborn ruminant may provide a unique opportunity to manipulate the symbiotic microbiota for a long-lasting impact in the adult ruminant (Yanez-Ruiz et al., 2015).

Colonisation: from birth (preruminant) to a fully functional rumen

Recent reviews describe the microbial community successions that occur in the rumen from birth to weaning and after, when animals feed exclusively on solid feeds (Malmuthuge et al., 2015, YanezRuiz et al., 2015, Meale et al., 2017a). Functional populations, as well as taxa present in adult rumens, appear very early after birth, in a progressive way and a defined sequence. Several studies monitored the establishment of the rumen bacterial community in calves from birth to weaning using high throughput sequencing and qPCR approaches (Jami et al., 2013, Rey et al., 2014, Guzman et al., 2015). They show that rapid changes occur in the composition of the rumen bacterial community during the first days of life. Proteobacteria and Streptococcus-related sequences are proportionally abundant in 1 to 3-day old calves and are rapidly replaced by strictly anaerobic bacterial taxa (Jami et al., 2013). Proteobacteria are then gradually replaced by Bacteroidetes as the 
animal grows, Firmicutes being present from early age to adulthood (Table 1). These results are in accordance with early studies using culture techniques reporting that aerobic and facultative anaerobic bacteria establish first (Fonty et al., 1987). Notwithstanding, strict anaerobes that are important for function in the mature rumen, such as cellulolytic bacteria and methanogenic archaea are already present in the rumen 1 or 2 days after birth (Fonty et al., 1987, Gagen et al., 2012, Jami et al., 2013, Guzman et al., 2015). Methanogenic archaea can be enumerated in the immature rumen of lambs at 2-4 days, well before the consumption of solid feeds, and after two weeks, their concentration is equivalent to that found in adult animals (Fonty et al., 1987, Morvan et al., 1994). Although not detected by culture, a low-abundant but diverse population of methanogens (predominantly Methanobrevibacter spp.) was identified using molecular methods in lambs placed into sterile isolators $17 \mathrm{~h}$ after birth (Gagen et al., 2012). A recent study in goat kids also indicated that active methanogens colonized the rumen at one day of life, Methanobrevibacter, Methanosphaera (both Methanobacteriales order) and Candidatus Methanomethylophilus (Methanomassiliicoccales order) being the top three genera (Wang et al., 2017b). There are four major methanogenic orders usually found in the rumen: Methanobacteriales, Methanomicrobiales, Methanosarcinales and Methanomassiliicoccales (Janssen and Kirs, 2008). All these are abundantly present in calves from day 1 to 2 weeks of age, whereas only Methanobacteriales and Methanomassiliicoccales could be qPCR-detected in the mature rumen (Friedman et al., 2017). Based on substrate utilisation for methanogenesis, the authors suggest that the early methanogenic community may be characterized by a high activity of methylotrophic methanogenesis, likely performed by members of the order Methanosarcinales. Eukaryotic microorganisms also establish sequentially. Anaerobic fungi can be enumerated in the rumen of lambs by 8-10 days after birth (Fonty et al., 1987). Anaerobic fungi, which are cellulolytic, are thus present in the rumen long before the animal ingests solid feeds regularly. Ciliates are detected from 2 to 3 weeks of age, with Entodinium establishing first (15-20 days), then Polyplastron, Eudiplodinium, and Epidinium (20-25 days), and finally Isotricha (50 days) (Fonty et al., 1988). In contrast to bacteria and archaea, protozoa do not establish when newborns are isolated from their dams shortly after birth (Fonty et al., 1988; Chaucheyras-Durand et al, personal communication). In addition, ciliate protozoa require the presence of a complex microbiota to establish (Fonty et al., 1983, Fonty et al., 1988). Figure 1 shows the main colonization events by groups of microbes in lamb's rumen throughout the suckling period and up to the end of weaning.

Colonization of the rumen wall by epimural bacteria is also age-related, with sequential diversification of bacterial morphotypes (Rieu et al., 1990). The phylum Proteobacteria is dominant on the rumen epithelium with an important contribution of the genus Escherichia (Jiao et al., 2015, 
Wang et al., 2017a). As for the lumen, the abundance of Proteobacteria associated with rumen epithelium decreases, and that of Firmicutes and Bacteroidetes increases with age (Jiao et al., 2015).

Large differences between digesta and epimural bacterial communities have been observed in the rumen of pre-weaned calves, with higher abundances of Prevotella and lower abundances of Bacteroidetes in digesta compared with epimural bacteria (Malmuthuge et al., 2014).

Although the focus of this review is on the rumen compartment, we will mention some aspects of the microbiota of other GIT sections when relevant and/or available. Whilst the rumen microbial community evolution with age has been well studied, information on the other pre-gastric compartments (reticulum and omasum) is scarce. Recent studies analysed fluid samples from these organs in goats from 3 to 56 days after birth (Lei et al., 2018) or in calves from birth to 21 days (Yeoman et al., 2018). As for the rumen, Proteobacteria gradually decrease with age while the relative abundance of Bacteroidetes increases.

For the post-gastric compartments, a surprisingly diverse microbiota is also described in the first hours post-delivery (Alipour et al., 2018). Firmicutes, Proteobacteria, Actinobacteria and Bacteroidetes dominate the newborn's rectal microbiota but composition rapidly changes in the early postnatal life. Pioneer studies reported E. coli and Streptococcus to be the firsts to colonise all GIT regions in calves and lambs few hours after birth (Smith, 1965) and culture-independent studies confirmed their high abundance in rectal microbiota one day after birth (Alipour et al., 2018). As for the rumen (Jami et al., 2013), it is assumed that these facultative anaerobes scavenge oxygen and render the environment suitable for strictly anaerobic gut microbes. Lactobacilli take advantage of these conditions and colonise all intestinal sections of one-day-old ruminants (Smith, 1965). Proteobacteria, Firmicutes and Bacteroidetes are the prevalent phyla in all anatomical locations within the first 3 weeks of life (Malmuthuge et al., 2014, Alipour et al., 2018, Yeoman et al., 2018). Firmicutes dominate distal parts of the GIT in young ruminants (colon and faeces), whereas Bacteroidetes abundance is higher in the reticulum, rumen, omasum and abomasum (Malmuthuge et al., 2014, Yeoman et al., 2018). It should be noted that while Firmicutes and Bacteroidetes are more abundant in luminal contents, Proteobacteria dominate mucosal samples (Yeoman et al., 2018). This niche specialisation for mucosa-associated populations is certainly driven by environmental conditions (presence of trace oxygen) and available substrates such as mucins. Interestingly, richness and $\alpha$-diversity in luminal samples increase with age in most anatomical sections, whereas no such trend is observed for mucosa-associated populations. Nevertheless, mucosa-associated bacterial communities in the small and large intestine are more diverse than digesta-associated communities (Malmuthuge et al., 2014). Though the post-gastric intestinal tract has not received much attention this far, there is evidence that its microbiota plays a crucial role in 
older animals' health and performance. For instance, lactic acid bacteria increase IgA production by stimulating host's adaptive immune system, boosting calves' active immunity by the time when passive immunity from colostrum decline (Corthésy et al., 2007).

\section{Interaction host-microbiota}

The gastrointestinal tract of mammals has a diverse array of non-specific and specific protective mechanisms to allow it to coexist with resident microbiota (Hooper et al., 2012). The functions of nutrients absorption, symbiotic microbial tolerance and pathogenic microbial barrier, create a conflict in function which requires a complex system of physical, biochemical and cellular mechanisms for protecting the gastro-intestinal epithelium and the host against invading agents (Kuhn and Stappenbeck, 2013). Several studies have shown the education process that the immune system needs to go through to deal with microbial loads and this is of particular relevance during early life stages (Collado et al., 2012, Wu and Wu, 2012). However, the mechanisms involved in the 'tolerance' to the first colonizers of the rumen are largely unknown, partly because the rumen epithelial structure has its own peculiarities as compared to the lower intestinal tract. The rumen has a multi-strated, keratinized epithelium with up to 15 cells layer and no organized lymphoid tissue (Sharpe et al., 1975), which limits the permeability of large molecules such as immunoglobulins. The immunological equilibrium in the rumen is thought to be achieved by a combination of two main mechanisms: i) the signalling of Toll-like receptors (TLRs) (Malmuthuge et al., 2012) and ii) provision of immunoglobulins (mainly secretory IgA) via saliva (Williams et al., 2009).

i) TLRs are a family of pattern recognition receptors (PRRs) that play a key role at mucosal surfaces by acting as sensors to detect molecular patterns expressed by both pathogenic and commensal microbiota alike. Cattle express a similar repertoire of TLRs compared with other mammalian species, as TLR1-10 have been identified and characterized (McGuire et al., 2006) and several studies have shown the relationship between nutritional disturbances (i.e. acidosis) and TLR expression levels. (Chen et al., 2012). However, little is known in relation to early-life colonization. The expression of TLR in the GIT of newborn calves is greater than at 6 months of age with the exception of TLR-1 and TLR-3 (Malmuthuge et al., 2012). More recently, Abecia et al. (2017) evaluated the impact of two different management practices in the early life of goat kids (maternal vs. artificial milk feeding) on the immune response of the rumen. They observed that TLRs $1,2,5,8$ and 10 displayed an age-dependent expression, consisting of increased gene expression between days 5 and 7 of life and then a subsequent decrease and stabilization. The increase in expression coincided with a rise in VFA concentration and microbial biomass colonization. However, although colonization continued in the following days, this did not trigger higher expression levels. This is in agreement with reports showing down regulation of TLR expression levels in the blood of newborns 
with increasing age, whereas memory T cells increased in number (Teran et al., 2011, Malmuthuge et al., 2012). The down-regulation of TLR activity might also be promoted by the production of butyrate that enhanced barrier function through stable tight junctions signalling (Jiao et al., 2017). Therefore, the few available published studies suggest that down-regulation with increasing age might be one mechanism by which the host avoids unnecessary inflammatory responses to commensal microbiota. In suckling calves fed raw milk, the expression of IL-8, IL-10 and claudin-4 in the mucosa of the colon was low compared to calves of the same age fed heat-treated milk (Bach et al., 2017). These calves fed raw milk had a higher Lactobacilli population suggesting that the down-regulation observed with increasing age mentioned above is associated with a more developed microbiota.

ii) Immunoglobulins find their way into the rumen mainly through saliva, particularly IgA that is the major Ig secreted in saliva and also the dominant type in the rumen (Subharat et al., 2015, Abecia et al., 2017). IgA is apparently more resistant to degradation in the rumen compared to IgG, possibly because the secretory component of IgA makes the immunoglobulin more resistant to proteases (Snoeck et al., 2006). Secretory IgA coats commensal bacteria in the GIT of calves (Tsuruta et al., 2012, Fouhse et al., 2017) and the oral microbiota coated with IgA had a greater resemblance to whole rumen microbiota than non-coated oral microbiota, suggesting salivary IgA-coating may be a host-derived mechanism impacting commensal colonization (Fouhse et al., 2017). This contrasts somehow with earlier works conducted on specific rumen microbes for the development of vaccines, which suggest that salivary IgA inhibit certain microbes. However, a recent work supports the hypothesis of Fouhse et al. (2017) by showing that specific immune recognition by IgA facilitated bacterial adherence to intestinal epithelial cells that mediates stable colonization (Donaldson et al., 2018). This suggests that in addition to its role in pathogen clearance, IgA can drive commensal microbes acceptance for colonization. This, however, remains to be demonstrated in early life colonization of the rumen.

Colonization of the rumen induces also a liver response with transcription of genes that can be associated with the development of tolerance to symbiotic microbes (Li et al., 2019). Following the transfer of rumen fluid from an adult cow into lactating calves, the main up-regulated genes in the host's liver were linked to immune response, anti-inflammatory response and cell signalling (Li et al., 2019).

\section{Weaning and stabilization of the rumen microbiota}

Important questions addressed in studies on the developing rumen are what are the main driving factors for a healthy and functional microbiota at weaning (age, pre-weaning diet, late weaning) and at what age the rumen microbiota stabilize and can be considered mature? The weaning transition 
under standard farm management practices could be a stressful period that reduces intake and growth. The developing rumen of a preweaned calf contains the same dominant phyla, Bacteroidetes, Firmicutes, and Proteobacteria, as the postweaned rumen, although the relative abundances of these phyla vary with age and stage of development (Li et al., 2012, Jami et al., 2013, Meale et al., 2016, Dias et al., 2018). Generally, the relative abundance of Firmicutes increases after weaning, while that of Bacteroidetes declines (Jami et al., 2013, Meale et al., 2016, Meale et al., 2017b). However, the precise evolution with time of these phyla as well as the successions of bacterial families or genera may be different depending on the type of ruminant and there are even differences between studies. The microbial successions also fluctuate depending on management practices, age at weaning or the nature of the feed (Meale et al., 2016, 2017a, 2017b). For example, $\beta$-diversity of ruminal microbiota shifted rapidly in early-weaned calves ( 6 weeks), whereas, a more gradual shift was observed in late-weaned calves (8 weeks) (Meale et al., 2017b). The authors concluded that the gradual increase in solid feed consumption of late-weaned calves resulted probably in lesser physiological stress during the weaning transition than that observed in earlyweaned calves. Also, feeding starter concentrate in addition to milk to pre-weaned calves promoted greater diversity of bacterial taxa known to degrade readily fermentable carbohydrates in the rumen (e.g., Megasphaera, Sharpea, and Succinivribrio) and favoured Methanosphaera instead of Methanobrevibacter (Dias et al., 2018). In this last study, the relative abundance of fungi did not change significantly with diet or age, maybe due to high inter-animal variation and low fibre content of the diet. In goats, recent work showed that the rumen microbial community and the metabolome before and after weaning are clearly different, illustrating the impact of the diet (Abecia et al., 2018). Also in goats, variability and instability in the composition of the methanogen community were observed according to the change of diet and age, and stabilization appears to occur at weaning (Wang et al., 2017b). Weaning seems to be a turning point for the rumen with a microbiota structure that resembles that of adult ruminants. Notwithstanding, at lower taxonomical level many microbial taxa differed (Dill-McFarland et al., 2017). The transition from weaning to an adult-like microbiota between weaning can take several months and up to one year of age (Fonty et al., 1988, DillMcFarland et al., 2017).

\section{Microbial activities and functions}

Most studies to date have used 16S (18S/ITS) rDNA amplicon sequencing for monitoring the rumen microbial colonization. It is important to complete these studies by measuring microbial activities or enumerating functional microbial species to ascertain the establishment of feeddegrading and other functional activities. As already mentioned, cellulolytic bacteria and methanogens are present in the rumen of 1 to 2-day old ruminants (Fonty et al., 1987, Guzman et al., 
2015). Enzymatic activities critical for utilisation of feed nutrients such as plant fibre, proteins and starch are detected in the rumen from the first days of life (Rey et al., 2012, Jiao et al., 2015). These activities increase throughout the first weeks showing maximal specific activity at around one month of age, which is after the initial stage of microbial colonization is achieved (Jiao et al., 2015). These changes may partially reflect dietary transitions from colostrum to milk or milk replacer, and then to a progressive consumption of solid feeds. As indicated above, methanogenic activity can be measured as early as 2-3 days of life (Morvan et al., 1994, Friedman et al., 2017). The establishment of hydrogenotrophic activity in the rumen of lambs, investigated by culture and isotope-labelling experiments, appeared also to be sequential. Hydrogen-dependent acetogenesis was present in the rumen of 20-h-old lambs while hydrogenotrophic methanogenesis was detected from 30-h of life. Hydrogen-utilizing sulphate-reducing bacteria established by the third day after birth (Morvan et al., 1994). The colonization of reductive acetogens after birth was also explored analysing the presence of genes acetyl-CoA synthase and formyltetrahydrofolate synthetase in the immature lamb rumen. Potential acetogens identified were affiliated with the Blautia genus and the Lachnospiraceae family, whereas establishment of methanogenic activity did not substantially affect acetogen diversity in these lambs (Gagen et al., 2012). The functional diversity of the rumen microbiome of the preruminant calf was also explored through metagenomics (Li et al., 2012). More than 8000 putative Pfam protein families were detected in the rumen of 14-day old calves with up to sixty glycosyl hydrolase families identified, indicating a high metabolic potential for carbohydrate processing long before weaning (Li et al., 2012). An increase in carbohydrate metabolism from 5 to 9 weeks of age in calves inferred from 16S rRNA gene sequences was also suggested (Meale et al., 2017b), although the use of functional metabolic predictions based on taxonomic information should be interpreted with prudence (Vieira-Silva et al., 2016).

\section{Modes of transmission}

It has been conventionally considered that the uterus is a sterile environment and microbial inoculation of the newborn's gut starts immediately after birth, through the vaginal canal, faecal material, colostrum, skin and saliva of the dam as well as from the environment. However, bacterial and archaeal DNA has been detected in the rumen, intestines and meconium of calves as early as 20 min after birth (Guzman et al., 2015), which led the authors to suggest that inoculation of the rumen may occur before birth. Studies on human and on mice newborns suggest that colonization of the intestine in these species starts in utero (Ihekweazu and Versalovic, 2018). This has led to investigations in ruminants with contrasting results. Some studies suggest that the colonization of the ruminant intestine may begin before birth (Alipour et al., 2018) but negative results were reported by others (Malmuthuge and Griebel, 2018). Caution should be exerted when interpreting 
results from humans or other animals as the structure of the placenta and the maternal-fetal interaction differ greatly among mammals, with ruminants having the most complete type of placental barrier (Benirschke et al., 2012). Another caveat is contamination when analysing this kind of samples with low microbial numbers (Malmuthuge and Griebel, 2018). The information available is not enough to confirm or disprove the in utero transfer of microbes into the gut in ruminants.

Whether or not the bacterial colonization of the digestive tract begins before birth, the relative contribution of all the other sources of inoculation is not well known. It is well established that the dam is an important source of microbial inoculation of the newborn rumen. Indeed, in newborns lambs separated from their dams and reared individually, no cellulolytic bacteria, fungi and protozoa established in the rumen (Fonty et al., 1988). Also, similarities between rumen methanogen populations found in newborn twin lambs suggested that the dam was the most important source of inoculation (Skillman et al., 2004). The dam's oral microbiota may be particularly important because of rumination, which would facilitate the oral transfer of rumen microorganisms to the newborn as a result of the natural instinct to lick them after birth. Colostrum and milk are also a source of bacterial colonization of the newborn gut. As for monogastric animals, liquid feeds in suckling ruminants go directly to the true stomach, the abomasum, through the oesophageal groove. However, the groove is not completely hermetic and small amounts of milk are also found in the rumen where taxa able to utilize milk nutrients such as Bacteroides and Lactobacillus are dominant in suckling calves fed milk only (Dias et al., 2018). The ability to grow on milk lactose by $F$. succinogenes can explain the active presence of this cellulolytic bacterium in pre-weaning calves (Ghali et al., 2017). The importance of the microbiota from different body sites of the dam to seed the newborn's gastrointestinal tract was explored recently (Alipour et al., 2018, Yeoman et al., 2018). The oral microbiota of the dam most closely resembled the rectal microbiota of the newborn calf in the study of Alipour et al. (2018). Whereas, in the study of Yeoman et al. (2018) the udder skin microbiota of the dam shared the largest number of OTUs with both luminal and mucosal microbiota of the calves from day 1-21 as compared with colostrum or vaginal samples. Surprisingly, fibrolytic bacteria and methanogenic archaea sequences were identified in the cow vaginal samples, suggesting a colonization role during birth (Yeoman et al., 2018). This is in addition to the important role that the dam vaginal microbiota has in colonizing the upper respiratory tract of the offspring (Lima et al., 2019).

\section{Impact of management practices}

Assuming that a key turning point in rumen microbial colonization is the introduction of solid feed in the diet (see above), an important issue to address is whether the feeding management of the newborn alters the colonization pattern. Few studies have compared the microbial colonization 
of the undeveloped rumen in the context of the factors that facilitate (or prevent) the colonization of some microbial groups (i.e. maternal influence, offspring reared in isolation, use of microbial modulators, etc).

As presented above, the dam has an important influence in early colonization. The maternal influence in relation to management systems has been further addressed in recent studies (Abecia et al., 2014b, Belanche et al., 2015, Abecia et al., 2017, 2018, Yeoman et al., 2018). Two main systems exist for rearing offspring in ruminant production. In commercial dairy systems, newborns are typically separated from the dam after birth and fed either milk replacer or whole milk. In contrast, in beef and extensive production systems, the offspring remains with the dam until weaning. These two systems, therefore, imply differences in regards to milk type (whole milk vs. milk replacer) and contact or not with adult conspecifics that in many cases are confounded. Protozoa in artificially reared animals showed a different colonization pattern as compared to those raised by the dams (Abecia et al., 2014a, Belanche et al., 2015). Natural milk feeding via the dam vs. artificial feeding with milk replacer resulted in consistently lower $\mathrm{pH}$ in the developing rumen of goat kids that stayed with the mothers (Abecia et al., 2014a). The authors hypothesised that naturally raised kids would have consumed more concentrate at an earlier stage as a result of social feeding learning as also shown by De Paula Vieira et al. (2012), who observed that the presence of an older companion with preweaned calves stimulated feeding behaviour and growth before and after weaning. In addition to learning behaviour, the presence of older conspecifics influences the rumen microbiota as damreared goat kits showed substantially greater bacterial diversity throughout the colonization process than those artificially reared (Abecia et al., 2017). Fibrobacteres (one of the main cellulolytic phyla in the rumen) was present in naturally raised kids from day seven after birth; however, it did not colonize the rumen of kids kept in isolation from dams until day 28 , similarly to Succiniclasticum which ferment succinate to propionate. Also, greater VFA concentration was observed in the rumen of kids with dams, which may suggest a quicker establishment of microbial fermentation in these animals (Abecia et al., 2014a). However, it remains unknown whether this distinct microbial colonization between animals, reared naturally or artificially, may have effects on the animal digestive performance later in life. The effect of the dam in the colonisation after birth deserves further research as it could help to develop new inoculation strategies in livestock farming.

\section{Modulating the gastrointestinal microbiota in young ruminants for health and production}

As stated in previous sections, a number of factors influence the GIT microbial colonization in young ruminants. Among them, the type of production system, farm management and diet are the 
most important. Good management practices normally recommended to reduce the onset of diseases and promote a vigorous growth in the first weeks of life have an obvious impact on the GIT microbiota. For instance, feeding high-quality colostrum allows establishing a beneficial, mucosaassociated bacterial barrier and thus provides protection against enteric infections in young ruminants (Malmuthuge et al., 2015). Colostrum favours the development of beneficial Bifidobacterium whereas it reduces potential pathogens and members of Escherichia and Shigella genera in the colon (Song et al., 2019). Suckling from the dam and contact with conspecific adults is the best way to ensure the natural acquisition of a functional GIT microbiota in early life. However, this otherwise natural practice is not always applicable under current production systems and nutritional interventions in early-life may be considered to facilitate (or prevent) the colonization of some microbial groups. In some particular cases, contact with the dam might not be recommended if there is a risk of transmission of non-desirable microbial populations. The risk of transmission of Mycobacterium avium subsp. paratuberculosis, the causative agent of Johne's disease, increased with the number of days that newborns remained with affected adults, particularly in the first week of life (Burgess et al., 2018). Inversely, it is reasonable to hypothesize that the contact with some animals should be promoted for facilitating the transmission of microbiotas associated to desired phenotypes such as reduced enteric methane emission or improved feed efficiency (Shabat et al., 2016, Difford et al., 2018).

Diet is a well-known modulator of the GIT microbiota at all ages but it seems to have a particular influencing role in the development of the microbiota and the microbiota-host dialogue in young ruminants (i.e.:Bach et al., 2017, Dill-McFarland et al., 2019, Dong et al., 2019). Differences in milk processing that can be considered minor from a macronutrient standpoint had large consequences on some gut microbial populations and inflammation parameters. As opposed to heat-treated milk (pasteurised and UHT), raw milk increased Lactobacillus numbers, decreased the expression of proinflammatory interleukins, and improved growth in dairy calves (Bach et al., 2017).

In addition to the management and diet factors cited, two main nutritional interventions in earlylife may be considered to modulate the GIT microbiota. These are the direct inoculation of specific microorganisms and the use of compounds (i.e. additives) that prevent or facilitate the colonization of some microbial groups. These two strategies are mainly tested for improving phenotypes of interest.

\section{Early-life strategies for improving health and production (case studies)}

Feeding live microorganisms to ruminants is not a novel concept and extensive work has been published on the use of direct-fed microbials (DFM) (Martin and Nisbet, 1992, Jeyanathan et al., 
2014). Theodorou et al. (1990) reported that the addition of an anaerobic rumen fungus, Neocallimastix spp., increased intake and live weight gain in calves at weaning, whilst (Ziolecka et al., 1984, Ziolecki et al., 1984) reported that a stabilized rumen extract enhanced live weight gain and stimulated rumen development in calves during weaning. The use of fresh rumen fluid inoculated in early life has gained new attention. Most recent studies showed that inoculation of fresh rumen fluid from adults into the rumen of newborns has a positive effect on productive parameters (Zhong et al., 2014, De Barbieri et al., 2015b). After weaning, average daily gain, intake, digestibility and rumen development improved in treated animals (lambs and kits) as compared to non-treated. Some of these effects may not last, although the composition of ruminal bacterial communities still differed up to 5 months of age, well after the initial inoculation (De Barbieri et al., 2015a, 2015b).

For specific DFM preparations, the administration of lactic acid bacteria to suckling calves was shown to stimulate rumination and ruminal development (Nakanishi et al., 1993) and the use of lactic acid bacteria or Bifidobacterium increased feed conversion efficiency and body weight gain (Abe et al., 1995). In this latter work, the DFMs decreased diarrhoea. The effect of lactic acid bacteria DFM on the prevalence of diarrhoea was confirmed in a meta-analysis (Signorini et al., 2012). Interestingly, the protection was only observed with multi-strains DFM and for calves fed raw milk. All these effects of lactic acid bacteria can be partially ascribed to the positive modulating activities of these bacterial groups on host immunology as described in the subsection Interaction host-microbiota (see above). The rumen bacterium Megasphaera elsdenii uses lactate to produce butyrate, a beneficial energy compound used by the GIT epithelial mucosa. This bacterium was also used as DFM in young ruminant with contrasting results. Muya et al. (2015) showed improved feed intake and rumen development, suggesting increased epithelium metabolism and improved absorption of digestive end products. Whereas, no effect was observed in another study using the same strain and a similar protocol (Yohe et al., 2018).

Diarrhoea is one of the main health issues in preweaned ruminants, particularly in dairy (USDA, 2018). A low faecal microbial diversity, notably a low relative abundance of Faecalibacterium spp., a symbiotic bacterium with known anti-inflammatory activity, was associated with an increased incidence of diarrhoea (Oikonomou et al., 2013). Inversely, Faecalibacterium spp. is positively associated with weight gain. Substantiating these effects, the use of Faecalibacterium prausnitzii as DFM in dairy calves decreased the incidence of severe diarrhoea by half and mortality by two thirds as compared to untreated controls (Foditsch et al., 2015).

Another strategy gaining attention is the use of life yeasts in early life. Although yeasts are widely used in ruminant nutrition (Chaucheyras-Durand et al., 2012), the concept of applying them in the diet of pre-ruminants to alter microbial colonization is more novel. It has been showed that 
yeasts, particularly as DFM, have positive effects on growth, rumen and small intestine development, immunity and general health of the calf (Kim et al., 2011, Alugongo et al., 2017). Specifically, Saccharomyces cerevisiae yeast (SCY) can improve DMI, growth, feed efficiency (Lesmeister and Heinrichs, 2004, Alugongo et al., 2017) and reduce diarrhoea in calves (Galvao et al., 2005, Brewer et al., 2014). Furthermore, subtle improvements were seen in rumen fermentation (increased butyrate production) and rumen papillae growth (Lesmeister and Heinrichs, 2004). These positive results, however, are more pronounced in calves stressed or exposed to significant levels of disease-causing agents. Despite the increasing number of published works studying the use of SCY in early-life of ruminants, there is a lack of knowledge about its impact on the microbial colonization. Recently, Terré et al. (2015) reported that supplementing young male calves with live SCY increased the presence of the fibrolytic bacterium $R$. albus in the rumen and it also increased rumen $\mathrm{pH}$ but no effect was observed on other bacteria and protozoa. Supplementation of newborn lambs fed milk replacer by live yeasts was also shown to increase the colonization of the rumen by F. succinogenes, fungi and protozoa (Chaucheyras-Durand et al, personal communication). Other reported effects of SCY are an increase in Lactobacillus in faeces (Fomenky et al., 2017) and greater oxidative burst and phagocytosis activity during weaning stress indicating a stronger innate immune response (Fomenky et al., 2018). Generally, for all DFM and the engraftment of adult rumen microbiota, there is still a need for further research in whole microbiome analysis and areas such as gut immunity and the persistence of effects later in life, especially in dairy systems due to their management of newborns. This information is necessary to draw consistent conclusions on their potential in early life.

\section{Early-life strategies for reducing enteric methane emissions (case studies)}

Modulating the microbial colonization in the developing rumen towards communities that produce less methane is also gaining attention. For this objective, compounds used as feed additives or feed supplements have been tested with two different approaches: one that specifically targets methanogens and the other uses general inhibitors that were described as having various mechanisms of action. For the specific approach, the application of bromochloromethane to young goat kids modified archaeal colonisation of the rumen, which was linked to a reduction in methane emission of around $50 \%$. The effects persisted for 3 months after weaning and cessation of treatment in kids raised by does that received the same treatment as the kids (Abecia et al., 2013, 2014a). For the generalist modulation approach, different types of lipids and plant extracts with proven efficacy to alter microbial metabolism and methane emissions in adult animals were tested. Debruyne et al. (2018) tested in goats prenatal and postnatal supplementation with coconut oil medium chain fatty acids. The treatment reduced in vitro methane emissions in four-week-old kids 
by reducing methanogen abundance and activity but at the expense of rumen fermentation and eubacterial abundance. Unfortunately, the treatment also suppressed daily gain of treated kids. Some rumen papillae characteristics differed at 28 weeks old due to postnatal treatment which ended at week 11 of life, indicating rumen papillary development can be affected by the early-life nutritional management. Saro et al. (2018) tested a combination of garlic oil and linseed oil in earlylife of lambs and the potential impact of re-treating the animals later in life. The archaeal methanogenic community was modified during the treatment but, in accordance with methane emissions, disappeared afterwards. In contrast, bacterial community structure differed between treated and non-treated lambs during and after the intervention. Rumen and urine metabolomics profiles conducted after the intervention ceased, highlighted interactions between microbes and metabolites, notably that of methylated compounds and Methanomassiliicocceae methanogens. They demonstrated that a long-term early-life intervention induced modifications in the composition of the rumen bacterial community that persisted after the intervention ceased with little or no effect on archaeal and protozoal communities. However, there was no persistence of the early-life intervention on methanogenesis suggesting a notable resilience for this function. Similarly, Lyons et al. (2017) treated lambs with linseed oil and found persistence of differences in the bacterial community structure but not associated changes in phenotypic response.

Methanogens occupy a specific niche in the rumen ecosystem. When methanogens are inhibited, there is a shift in hydrogen flow and other electron-donor metabolites towards alternative electron acceptors such as propionate. This implies the rearrangement of trophic networks and changes in microbial populations that are mainly observed for the bacterial communities in all studies cited above. The abundance of methanogens was not always affected but their diversity was commonly altered although the changes were different probably due to the type of inhibitor used. Linseed and garlic oil decreased the Methanomassiliicoccales relative abundance in lambs (Saro et al., 2018). Bromochloromethane increased the relative abundance of Methanobrevibacter and decreased that of Methanosphaera in kids (Abecia et al., 2014b). Whereas, linseed increased Methanosphaera relative abundance in lambs (Lyons et al., 2017).

\section{Summary and outstanding questions/future trends}

From birth, ruminants are constantly exposed to different sources of microbes. The establishment of stable communities depends on the creation of suitable conditions with a close interplay between diet and maturation of the GIT. The sequential evolution of the gut microbiota that is followed by the expansion of metabolic functions is a coordinated process that matches the anatomical and physiological development of the GIT. In the non-ruminant phase (0-3 weeks), the 
establishment of a diverse microbial community, particularly bacteria in the post-gastric section, is important for health and grow (Oikonomou et al., 2013, Bach et al., 2017), whereas changes in the rumen are predominant during the transition (3-8 weeks) and rumination phases (+8 weeks of age) (Jiao et al., 2015). The periods when these alimentary, anatomical and physiological modifications occur seem to be the more suitable for interventions for long-term modifications of the GIT microbiota (Dill-McFarland et al., 2019).

The GIT microbiota at an early age is influenced and can be modulated by various strategies (Figure 2). Nevertheless, for the application of these strategies in farms there are still some outstanding questions that need to be addressed. Ongoing research on the role of GIT microbiota on animal phenotypes will bring information on the type of microbial communities that should be favoured in young ruminants. The effect of the prenatal period, including whether there is a prenatal colonisation of the GIT and its role in the establishment of the mature microbiota have to be assessed. Similarly, the best window(s) of age when the microbiota could be modulated have to be refined. Establishment of different populations might not be a single isolated event and a continuous exposure seems to be necessary for acquiring adult-like communities (Morgavi et al., 2015) or successfully engraft specific microbes (Yohe et al., 2018). A better definition of the role of early microbial communities on the host-microbiota dialogue and the regulation of the immune system is also important for this subject. Expected advances in all these topics in the coming years have the potential to improve the sustainability of ruminant production through better health in young animals and enhanced efficiency.

\section{Where to look for further information}

\section{Key conferences}

- Gut Microbiology Symposium; jointly organized by INRA France and the Rowett Institute Aberdeen University, every two years

- Congress on Gastrointestinal Function; in Chicago (USA) every two years

- Annual meetings from animal science societies such as Annual Meeting of the European Federation of Animal Science and American Dairy Science Association

- Smart Calve rearing Conference (https://smart-calf-rearing.com)

Major international research projects and networks

- Rumen Microbial Genomics Network, Global Research Alliance-Livestock Group. A large international network fostering collaborations and exchanges between microbiologists working with ruminants 
(https://globalresearchalliance.org/research/livestock/networks/rumen-microbial-genomicsnetwork/)

- MASTER (Microbiome Applications for Sustainable food systems through Technologies and EnteRprise) a H2020 project focusing on the characterisation and modulation of microbiomes from different environments including the GIT of ruminants (http://www.master-h2020.eu/)

\section{References}

Abe, F., N. Ishibashi, and S. Shimamura. 1995. Effect of Administration of Bifidobacteria and Lactic Acid Bacteria to Newborn Calves and Piglets. J. Dairy Sci. 78:2838-2846. 10.3168/jds.S00220302(95)76914-4

Abecia, L., E. Jiménez, G. Martínez-Fernandez, A. I. Martín-García, E. Ramos-Morales, E. Pinloche, S. E. Denman, C. J. Newbold, and D. R. Yáñez-Ruiz. 2017. Natural and artificial feeding management before weaning promote different rumen microbial colonization but not differences in gene expression levels at the rumen epithelium of newborn goats. PLOS ONE 12:e0182235. 10.1371/journal.pone.0182235

Abecia, L., A. I. Martín-García, G. Martínez, C. J. Newbold, and D. R. Yáñez-Ruiz. 2013. Nutritional intervention in early life to manipulate rumen microbial colonization and methane output by kid goats postweaning. J. Anim. Sci. 91:4832-4840. 10.2527/jas.2012-6142

Abecia, L., G. Martínez-Fernandez, K. Waddams, A. I. Martín-García, E. Pinloche, C. J. Creevey, S. E. Denman, C. J. Newbold, and D. R. Yáñez-Ruiz. 2018. Analysis of the rumen microbiome and metabolome to study the effect of an antimethanogenic treatment applied in early life of kid goats. Front. Microbiol. 9. 10.3389/fmicb.2018.02227

Abecia, L., E. Ramos-Morales, G. Martínez-Fernandez, A. Arco, A. Martín-García, C. Newbold, and D. R. Yáñez-Ruiz. 2014a. Feeding management in early life influences microbial colonisation and fermentation in the rumen of newborn goat kids. Anim. Prod. Sci. 54:1449-1454.

Abecia, L., K. E. Waddams, G. Martinez-Fernandez, A. I. Martin-Garcia, E. Ramos-Morales, C. J. Newbold, and D. R. Yanez-Ruiz. 2014b. An antimethanogenic nutritional intervention in early life of ruminants modifies ruminal colonization by Archaea. Archaea 2014:841463. 10.1155/2014/841463

Alipour, M. J., J. Jalanka, T. Pessa-Morikawa, T. Kokkonen, R. Satokari, U. Hynonen, A. livanainen, and M. Niku. 2018. The composition of the perinatal intestinal microbiota in cattle. jScientific Reports 8:10437. 10.1038/s41598-018-28733-y

Alugongo, G. M., J. X. Xiao, Z. H. Wu, S. L. Li, Y. J. Wang, and Z. J. Cao. 2017. Review: Utilization of yeast of Saccharomyces cerevisiae origin in artificially raised calves. J. Anim. Sci. Biotechnol. 8. 10.1186/s40104-017-0165-5

Bach, A., A. Aris, M. Vidal, F. Fàbregas, and M. Terré. 2017. Influence of milk processing temperature on growth performance, nitrogen retention, and hindgut's inflammatory status and bacterial populations in a calf model. Journal of Dairy Research 84:355-359. 10.1017/\$0022029917000401

Belanche, A., G. de la Fuente, and C. J. Newbold. 2015. Effect of progressive inoculation of fauna-free sheep with holotrich protozoa and total-fauna on rumen fermentation, microbial diversity and methane emissions. FEMS Microbiol. Ecol. 91. 10.1093/femsec/fiu026

Benirschke, K., G. J. Burton, and R. N. Baergen. 2012. Pathology of the Human Placenta. Springer Science \& Business Media. 
Brewer, M. T., K. L. Anderson, I. Yoon, M. F. Scott, and S. A. Carlson. 2014. Amelioration of salmonellosis in pre-weaned dairy calves fed Saccharomyces cerevisiae fermentation products in feed and milk replacer. Vet. Microbiol. 172:248-255. 10.1016/j.vetmic.2014.05.026

Burgess, T. L., C. L. Witte, and B. A. Rideout. 2018. Early-life exposures and Johne's disease risk in zoo ruminants. J. Vet. Diagn. Invest. 30:78-85. 10.1177/1040638717735350

Chaucheyras-Durand, F., E. Chevaux, C. Martin, and E. Forano. 2012. Use of Yeast Probiotics in Ruminants: Effects and Mechanisms of Action on Rumen pH, Fibre Degradation, and Microbiota According to the Diet. Pages 119-152 in Probiotic in Animals. E. Rigobelo, ed. Intechopen.

Chen, Y., M. Oba, and L. L. Guan. 2012. Variation of bacterial communities and expression of Toll-like receptor genes in the rumen of steers differing in susceptibility to subacute ruminal acidosis. Vet. Microbiol. 159:451-459. 10.1016/j.vetmic.2012.04.032

Collado, M. C., M. Cernada, C. Baüerl, M. Vento, and G. Pérez-Martínez. 2012. Microbial ecology and host-microbiota interactions during early life stages. Gut Microbes 3:352-365. 10.4161/gmic.21215

Corthésy, B., H. R. Gaskins, and A. Mercenier. 2007. Cross-talk between probiotic bacteria and the host immune system. J. Nutr. 137:781S-790S. 10.1093/jn/137.3.781S

Costello, E., K. Stagaman, L. Dethlefsen, B. Bohannan, and D. Relman. 2012. The application of ecological theory toward an understanding of the human microbiome. Science 336:1255-1262. $10.1126 /$ science. 1224203

De Barbieri, I., R. S. Hegarty, C. Silveira, L. M. Gulino, V. H. Oddy, R. A. Gilbert, A. V. Klieve, and D. Ouwerkerk. 2015a. Programming rumen bacterial communities in newborn Merino lambs. Small Ruminant Research 129:48-59. 10.1016/j.smallrumres.2015.05.015

De Barbieri, I., R. S. Hegarty, C. Silveira, and V. H. Oddy. 2015b. Positive consequences of maternal diet and post-natal rumen inoculation on rumen function and animal performance of Merino lambs. Small Ruminant Research 129:37-47. 10.1016/j.smallrumres.2015.05.017

De Paula Vieira, A., M. A. G. von Keyserlingk, and D. M. Weary. 2012. Presence of an older weaned companion influences feeding behavior and improves performance of dairy calves before and after weaning from milk. J. Dairy Sci. 95:3218-3224. d10.3168/jds.2011-4821

Debruyne, S., A. Ruiz-Gonzalez, E. Artiles-Ortega, B. Ampe, W. Van Den Broeck, E. De Keyser, L. Vandaele, K. Goossens, and V. Fievez. 2018. Supplementing goat kids with coconut medium chain fatty acids in early life influences growth and rumen papillae development until 4 months after supplementation but effects on in vitro methane emissions and the rumen microbiota are transient. J. Anim. Sci. 96:1978-1995. 10.1093/jas/sky070

Dias, J., M. I. Marcondes, S. Motta de Souza, B. Cardoso da Mata e Silva, M. Fontes Noronha, R. Tassinari Resende, F. S. Machado, H. Cuquetto Mantovani, K. A. Dill-McFarland, and G. Suen. 2018. Bacterial community dynamics across the gastrointestinal tracts of dairy calves during preweaning development. Appl. Environ. Microbiol. 84. 10.1128/aem.02675-17

Difford, G. F., D. R. Plichta, P. Lovendahl, J. Lassen, S. J. Noel, O. Hojberg, A. D. G. Wright, Z. G. Zhu, L. Kristensen, H. B. Nielsen, B. Guldbrandtsen, and G. Sahana. 2018. Host genetics and the rumen microbiome jointly associate with methane emissions in dairy cows. Plos. Genet. 14. 10.1371/journal.pgen.1007580

Dill-McFarland, K. A., J. D. Breaker, and G. Suen. 2017. Microbial succession in the gastrointestinal tract of dairy cows from 2 weeks to first lactation. Sci. Rep. 7:40864. 10.1038/srep40864

Dill-McFarland, K. A., P. J. Weimer, J. D. Breaker, and G. Suen. 2019. Diet influences early microbiota development in dairy calves without long-term impacts on milk production. Appl. Environ. Microbiol. 85:e02141-02118. 10.1128/aem.02141-18 
Donaldson, G. P., M. S. Ladinsky, K. B. Yu, J. G. Sanders, B. B. Yoo, W.-C. Chou, M. E. Conner, A. M. Earl, R. Knight, P. J. Bjorkman, and S. K. Mazmanian. 2018. Gut microbiota utilize immunoglobulin A for mucosal colonization. Science 360:795-800. 10.1126/science.aaq0926

Dong, L.-f., J.-n. Ma, Y. Tu, and Q.-y. Diao. 2019. Weaning methods affect ruminal methanogenic archaea composition and diversity in Holstein calves. J. Integr. Agric. 18:1080-1092. 10.1016/S20953119(18)62120-3

Foditsch, C., R. V. Pereira, E. K. Ganda, M. S. Gomez, E. C. Marques, T. Santin, and R. C. Bicalho. 2015. Oral administration of Faecalibacterium prausnitzii decreased the incidence of severe diarrhea and related mortality rate and increased weight gain in preweaned dairy heifers. PLoS One 10. 10.1371/journal.pone.0145485

Fomenky, B. E., J. Chiquette, N. Bissonnette, G. Talbot, P. Y. Chouinard, and E. M. Ibeagha-Awemu. 2017. Impact of Saccharomyces cerevisiae boulardii CNCMI-1079 and Lactobacillus acidophilus BT1386 on total lactobacilli population in the gastrointestinal tract and colon histomorphology of Holstein dairy calves. Anim. Feed Sci. Tech. 234:151-161. 10.1016/j.anifeedsci.2017.08.019

Fomenky, B. E., J. Chiquette, M. Lessard, N. Bissonnette, G. Talbot, Y. P. Chouinard, and E. M. Ibeagha-Awemu. 2018. Saccharomyces cerevisiae var. boulardii CNCM I-1079 and Lactobacillus acidophilus BT1386 influence innate immune response and serum levels of acute-phase proteins during weaning in Holstein calves. Can. J. Anim. Sci. 98:576-588. 10.1139/cjas-2017

Fonty, G., P. Gouet, J. P. Jouany, and J. Senaud. 1983. Ecological factors determining establishment of cellulolytic bacteria and protozoa in the rumens of meroxenic lambs. J. Gen. Microbiol. 129:213-223. 10.1099/00221287-129-1-213

Fonty, G., P. Gouet, J. P. Jouany, and J. Senaud. 1987. Establishment of the microflora and anaerobic fungi in the rumen of lambs. J. Gen. Microbiol. 133:1835-1843.

Fonty, G., J. Senaud, J. P. Jouany, and P. Gouet. 1988. Establishment of ciliate protozoa in the rumen of conventional and conventionalized lambs: influence of diet and management conditions. Can. J. Microbiol. 34:235-241.

Fouhse, J. M., L. Smiegielski, M. Tuplin, L. L. Guan, and B. P. Willing. 2017. Host immune selection of rumen bacteria through salivary secretory IgA. Front. Microbiol. 8:9. 10.3389/fmicb.2017.00848

Friedman, N., E. Jami, and I. Mizrahi. 2017. Compositional and functional dynamics of the bovine rumen methanogenic community across different developmental stages. Environ. Microbiol. 19:3365-3373. 10.1111/1462-2920.13846

Gagen, E. J., P. Mosoni, S. E. Denman, R. Al Jassim, C. S. McSweeney, and E. Forano. 2012. Methanogen colonisation does not significantly alter acetogen diversity in lambs isolated $17 \mathrm{~h}$ after birth and raised aseptically. Microb. Ecol. 10.1007/s00248-012-0024-z

Galvao, K. N., J. E. P. Santos, A. Coscioni, M. Villasenor, W. M. Sischo, and A. C. B. Berge. 2005. Effect of feeding live yeast products to calves with failure of passive transfer on performance and patterns of antibiotic resistance in fecal Escherichia coli. Reprod. Nutr. Dev. 45:427-440. 10.1051/rnd:2005040

Ghali, I., A. Sofyan, H. Ohmori, T. Shinkai, and M. Mitsumori. 2017. Diauxic growth of Fibrobacter succinogenes S85 on cellobiose and lactose. FEMS Microbiol. Lett. 364:9. 10.1093/femsle/fnx150

Guzman, C. E., L. T. Bereza-Malcolm, B. De Groef, and A. E. Franks. 2015. Presence of selected methanogens, fibrolytic bacteria, and proteobacteria in the gastrointestinal tract of neonatal dairy calves from birth to 72 hours. PLOS ONE 10:e0133048. 10.1371/journal.pone.0133048

Hooper, L. V., D. R. Littman, and A. J. Macpherson. 2012. Interactions between the microbiota and the immune system. Science 336:1268-1273. 10.1126/science.1223490 
Ihekweazu, F. D. and J. Versalovic. 2018. Development of the pediatric gut microbiome: impact on health and disease. Am. J. Clin. Nutr. 356:413-423. 10.1016/j.amjms.2018.08.005

Jami, E., A. Israel, A. Kotser, and I. Mizrahi. 2013. Exploring the bovine rumen bacterial community from birth to adulthood. ISME J 7:1069-1079. 10.1038/ismej.2013.2

Janssen, P. H. and M. Kirs. 2008. Structure of the archaeal community of the rumen. Appl. Environ. Microbiol. 74:3619-3625. 10.1128/aem.02812-07

Jeyanathan, J., C. Martin, and D. P. Morgavi. 2014. The use of direct-fed microbials for mitigation of ruminant methane emissions: a review. Animal 8:250-261. 10.1017/S1751731113002085

Jiao, J., X. Li, K. A. Beauchemin, Z. Tan, S. Tang, and C. Zhou. 2015. Rumen development process in goats as affected by supplemental feeding $v$. grazing: age-related anatomic development, functional achievement and microbial colonisation. Br. J. Nutr. 113:888-900. 10.1017/\$0007114514004413

Jiao, J., C. Zhou, L. L. Guan, C. S. McSweeney, S. Tang, M. Wang, and Z. Tan. 2017. Shifts in host mucosal innate immune function are associated with ruminal microbial succession in supplemental feeding and grazing goats at different ages. Front. Microbiol. 8:1655. 10.3389/fmicb.2017.01655

Kim, M. H., J. Y. Yang, S. D. Upadhaya, H. J. Lee, C. H. Yun, and J. K. Ha. 2011. The stress of weaning influences serum levels of acute-phase proteins, iron-binding proteins, inflammatory cytokines, cortisol, and leukocyte subsets in Holstein calves. Journal of Veterinary Science 12:151-157.

Kuhn, K. A. and T. S. Stappenbeck. 2013. Peripheral education of the immune system by the colonic microbiota. Semin. Immunol. 25:364-369. mo10.1016/j.smim.2013.10.002

Lei, Y., K. Zhang, M. Guo, G. Li, C. Li, B. Li, Y. Yang, Y. Chen, and X. Wang. 2018. Exploring the SpatialTemporal Microbiota of Compound Stomachs in a Pre-weaned Goat Model. Front. Microbiol. 9:1846. 10.3389/fmicb.2018.01846

Lesmeister, K. E. and A. J. Heinrichs. 2004. Effects of corn processing on growth characteristics, rumen development, and rumen parameters in neonatal dairy calves. J. Dairy Sci. 87:3439-3450. 10.3168/jds.S0022-0302(04)73479-7

Ley, R. E., C. A. Lozupone, M. Hamady, R. Knight, and J. I. Gordon. 2008. Worlds within worlds: evolution of the vertebrate gut microbiota. Nat. Rev. Microbiol. 6:776-788. 10.1038/Nrmicro1978

Li, R. W., E. E. Connor, C. Li, R. L. Baldwin Vi, and M. E. Sparks. 2012. Characterization of the rumen microbiota of pre-ruminant calves using metagenomic tools. Environ. Microbiol. 14:129-139. 10.1111/j.1462-2920.2011.02543.x

Li, W. L., A. Edwards, C. Riehle, M. Cox, S. Raabis, J. Skarlupka, A. Steinberger, J. Walling, D. Bickhart, and G. Suen. 2019. Transcriptomics analysis of host liver and meta-transcriptome analysis of rumen epimural microbial community in young calves treated with artificial dosing of rumen content from adult donor cow. Sci. Rep. 9. 10.1038/s41598-018-37033-4

Lima, S. F., M. L. d. S. Bicalho, and R. C. Bicalho. 2019. The Bos taurus maternal microbiome: Role in determining the progeny early-life upper respiratory tract microbiome and health. PLOS ONE 14:e0208014. 10.1371/journal.pone.0208014

Lyons, T., T. Boland, S. Storey, and E. Doyle. 2017. Linseed oil supplementation of lambs' diet in early life leads to persistent changes in rumen microbiome structure. Front. Microbiol. 8:1656.

10.3389/fmicb.2017.01656

Malmuthuge, N. and P. J. Griebel. 2018. Fetal environment and fetal intestine are sterile during the third trimester of pregnancy. Vet. Immunol. Immunopathol. 204:59-64.

10.1016/j.vetimm.2018.09.005 
Malmuthuge, N., P. J. Griebel, and L. L. Guan. 2014. Taxonomic identification of commensal bacteria associated with the mucosa and digesta throughout the gastrointestinal tracts of preweaned calves. Appl. Environ. Microbiol. 80:2021-2028. 10.1128/aem.03864-13

Malmuthuge, N., P. J. Griebel, and L. L. Guan. 2015. The gut microbiome and its potential role in the development and function of newborn calf gastrointestinal tract. Front. Vet. Sci. 2.

10.3389/fvets.2015.00036

Malmuthuge, N., M. Li, P. Fries, P. J. Griebel, and L. L. Guan. 2012. Regional and age dependent changes in gene expression of Toll-like receptors and key antimicrobial defence molecules throughout the gastrointestinal tract of dairy calves. Vet. Immunol. Immunopathol. 146:18-26. 10.1016/j.vetimm.2012.01.010

Martin, S. A. and D. J. Nisbet. 1992. Effect of direct-fed microbials on rumen microbial fermentation. J. Dairy Sci. 75:1736-1744. 10.3168/jds.S0022-0302(92)77932-6

McGuire, K., M. Jones, D. Werling, J. L. Williams, E. J. Glass, and O. Jann. 2006. Radiation hybrid mapping of all 10 characterized bovine Toll-like receptors. Anim. Genet. 37:47-50. 10.1111/j.13652052.2005.01364.x

Meale, S. J., F. Chaucheyras-Durand, H. Berends, L. L. Guan, and M. A. Steele. 2017a. From pre- to postweaning: Transformation of the young calf's gastrointestinal tract. J. Dairy Sci. 100:5984-5995. 10.3168/jds.2016-12474

Meale, S. J., S. Li, P. Azevedo, H. Derakhshani, J. C. Plaizier, E. Khafipour, and M. A. Steele. 2016. Development of ruminal and fecal microbiomes are affected by weaning but not weaning strategy in dairy calves. Front. Microbiol. 7:582. 10.3389/fmicb.2016.00582

Meale, S. J., S. C. Li, P. Azevedo, H. Derakhshani, T. J. DeVries, J. C. Plaizier, M. A. Steele, and E. Khafipour. 2017b. Weaning age influences the severity of gastrointestinal microbiome shifts in dairy calves. Sci. Rep. 7:198. 10.1038/s41598-017-00223-7

Moraïs, S. and I. Mizrahi. 2019. The road not taken: the rumen microbiome, functional groups, and community states. Trends Microbiol. 27:538-549. s10.1016/j.tim.2018.12.011

Morgavi, D. P., E. Rahahao-Paris, M. Popova, J. Boccard, K. F. Nielsen, and H. Boudra. 2015. Rumen microbial communities influence metabolic phenotypes in lambs. Front. Microbiol. 6.

10.3389/fmicb.2015.01060

Morvan, B., J. Dore, F. Rieu-Lesme, L. Foucat, G. Fonty, and P. Gouet. 1994. Establishment of hydrogen-utilizing bacteria in the rumen of the newborn lamb. FEMS Microbiol. Lett. 117:249-256.

Muya, M. C., F. V. Nherera, K. A. Miller, C. C. Aperce, P. M. Moshidi, and L. J. Erasmus. 2015. Effect of Megasphaera elsdenii NCIMB 41125 dosing on rumen development, volatile fatty acid production and blood beta-hydroxybutyrate in neonatal dairy calves. J. Anim. Physiol. Anim. Nutr. (Berl) 99:913918. 10.1111/jpn.12306

Nakanishi, Y., C. W. Arave, and P. H. Stewart. 1993. Effects of feeding Lactobacillus acidophilus yogurt on performance and behavior of dairy calves. J. Dairy Sci. 76:(Suppl. 1):244.

Oikonomou, G., A. G. V. Teixeira, C. Foditsch, M. L. Bicalho, V. S. Machado, and R. C. Bicalho. 2013. Fecal Microbial Diversity in Pre-Weaned Dairy Calves as Described by Pyrosequencing of Metagenomic $16 \mathrm{~S}$ rDNA. Associations of Faecalibacterium Species with Health and Growth. PLOS ONE 8:e63157. 10.1371/journal.pone.0063157

Rey, M., F. Enjalbert, S. Combes, L. Cauquil, O. Bouchez, and V. Monteils. 2014. Establishment of ruminal bacterial community in dairy calves from birth to weaning is sequential. J. Appl. Microbiol. 116:245-257. 10.1111/jam.12405 
Rey, M., F. Enjalbert, and V. Monteils. 2012. Establishment of ruminal enzyme activities and fermentation capacity in dairy calves from birth through weaning. J. Dairy Sci. 95:1500-1512. 10.3168/jds.2011-4902

Rieu, F., G. Fonty, B. Gaillard, and P. Gouet. 1990. Electron microscopy study of the bacteria adherent to the rumen wall in young conventional lambs. Can. J. Microbiol. 36:140-144.

Saro, C., U. M. Hohenester, M. Bernard, M. Lagrée, C. Martin, M. Doreau, H. Boudra, M. Popova, and D. P. Morgavi. 2018. Effectiveness of interventions to modulate the rumen microbiota composition and function in pre-ruminant and ruminant lambs. Front. Microbiol. 9. 10.3389/fmicb.2018.01273

Savage, D. C. 1977. Microbial ecology of the gastrointestinal tract. Annu. Rev. Microbiol. 31:107-133. 10.1146/annurev.mi.31.100177.000543

Shabat, S. K., G. Sasson, A. Doron-Faigenboim, T. Durman, S. Yaacoby, M. E. Berg Miller, B. A. White, N. Shterzer, and I. Mizrahi. 2016. Specific microbiome-dependent mechanisms underlie the energy harvest efficiency of ruminants. ISME J. 10.1038/ismej.2016.62

Sharpe, M., M. Latham, and B. Reiter. 1975. The immune response of the host animal to bacteria in the rumen and caecum. Digestion and Metabolism in the Ruminant (Ed) McDonald IW dan Warner, $\mathrm{ACl}$ 1st ed. The University of New England, Sydney p 149.

Signorini, M. L., L. P. Soto, M. V. Zbrun, G. J. Sequeira, M. R. Rosmini, and L. S. Frizzo. 2012. Impact of probiotic administration on the health and fecal microbiota of young calves: A meta-analysis of randomized controlled trials of lactic acid bacteria. Res. Vet. Sci. 93:250-258.

10.1016/j.rvsc.2011.05.001

Skillman, L. C., P. N. Evans, G. E. Naylor, B. Morvan, G. N. Jarvis, and K. N. Joblin. 2004. 16S ribosomal DNA-directed PCR primers for ruminal methanogens and identification of methanogens colonising young lambs. Anaerobe 10:277-285. 10.1016/j.anaerobe.2004.05.003

Smith, H. W. 1965. The development of the flora of the alimentary tract in young animals. J. Pathol. Bacteriol. 90:495-513. 10.1002/path.1700900218

Snoeck, V., I. R. Peters, and E. Cox. 2006. The IgA system: a comparison of structure and function in different species. Vet. Res. 37:455-467. 10.1051/vetres:2006010

Song, Y., N. Malmuthuge, F. Y. Li, and L. L. Guan. 2019. Colostrum feeding shapes the hindgut microbiota of dairy calves during the first $12 \mathrm{~h}$ of life. FEMS Microbiol. Ecol. 95:12.

10.1093/femsec/fiy203

Subharat, S., D. Shu, T. Zheng, B. M. Buddle, P. H. Janssen, D. Luo, and D. N. Wedlock. 2015. Vaccination of cattle with a methanogen protein produces specific antibodies in the saliva which are stable in the rumen. Vet. Immunol. Immunopathol. 164:201-207. 10.1016/j.vetimm.2015.02.008

Teran, R., E. Mitre, M. Vaca, S. Erazo, G. Oviedo, M. P. Hübner, M. E. Chico, J. J. Mattapallil, Q. Bickle, L. C. Rodrigues, and P. J. Cooper. 2011. Immune system development during early childhood in tropical Latin America: Evidence for the age-dependent down regulation of the innate immune response. Clinical Immunology 138:299-310. 10.1016/j.clim.2010.12.011

Terré, M., G. Maynou, A. Bach, and M. Gauthier. 2015. Effect of Saccharomyces cerevisiae CNCM I1077 supplementation on performance and rumen microbiota of dairy calves. Prof. Anim. Sci. 31:153-158. 10.15232/pas.2014-01384

Theodorou, M., D. Beever, M. Haines, and A. Brooks. 1990. The effect of a fungal probiotic on intake and performance of early weaned calves. Anim. Prod. 50:577.

Tsuruta, T., R. Inoue, T. Tsukahara, M. Nakamoto, H. Hara, K. Ushida, and T. Yajima. 2012.

Commensal bacteria coated by secretory immunoglobulin $A$ and immunoglobulin $G$ in the gastrointestinal tract of pigs and calves. Anim. Sci. J. 83:799-804. 10.1111/j.1740-0929.2012.01026.x 
USDA. 2018. Dairy 2014, "Health and Management Practices on U.S. Dairy Operations, 2014". Vol. \#696.0218. USDA-APHIS-VS-CEAH-NAHMS, Fort Collins, CO.

Vieira-Silva, S., G. Falony, Y. Darzi, G. Lima-Mendez, R. Garcia Yunta, S. Okuda, D. Vandeputte, M. Valles-Colomer, F. Hildebrand, S. Chaffron, and J. Raes. 2016. Species-function relationships shape ecological properties of the human gut microbiome. Nat. Microbiol. 1:16088.

10.1038/nmicrobiol.2016.88

Wang, Z., C. Elekwachi, J. Jiao, M. Wang, S. Tang, C. Zhou, Z. Tan, and R. J. Forster. 2017a. Changes in metabolically active bacterial community during rumen development, and their alteration by rhubarb root powder revealed by $16 \mathrm{~s}$ rRNA amplicon sequencing. Front. Microbiol. 8.

10.3389/fmicb.2017.00159

Wang, Z., C. O. Elekwachi, J. Jiao, M. Wang, S. Tang, C. Zhou, Z. Tan, and R. J. Forster. 2017b. Investigation and manipulation of metabolically active methanogen community composition during rumen development in black goats. Sci. Rep. 7:422. 10.1038/s41598-017-00500-5

Williams, Y. J., S. Popovski, S. M. Rea, L. C. Skillman, A. F. Toovey, K. S. Northwood, and A.-D. G. Wright. 2009. A vaccine against rumen methanogens can alter the composition of archaeal populations. Appl. Environ. Microbiol. 75:1860-1866. 10.1128/aem.02453-08

$\mathrm{Wu}, \mathrm{H}$.-J. and E. Wu. 2012. The role of gut microbiota in immune homeostasis and autoimmunity. Gut Microbes 3:4-14. 10.4161/gmic.19320

Yanez-Ruiz, D. R., L. Abecia, and C. J. Newbold. 2015. Manipulating rumen microbiome and fermentation through interventions during early life: a review. Front. Microbiol. 6.

10.3389/fmicb.2015.01133

Yáñez-Ruiz, D. R., B. Macías, E. Pinloche, and C. J. Newbold. 2010. The persistence of bacterial and methanogenic archaeal communities residing in the rumen of young lambs. FEMS Microbiol. Ecol. 72:272-278. 10.1111/j.1574-6941.2010.00852.x

Yeoman, C. J., S. L. Ishaq, E. Bichi, S. K. Olivo, J. Lowe, and B. M. Aldridge. 2018. Biogeographical differences in the influence of maternal microbial sources on the early successional development of the bovine neonatal gastrointestinal tract. Sci. Rep. 8:3197. 10.1038/s41598-018-21440-8

Yohe, T. T., B. D. Enger, L. Wang, H. L. M. Tucker, C. A. Ceh, C. L. M. Parsons, Z. Yu, and K. M. Daniels. 2018. Short communication: Does early-life administration of a Megasphaera elsdenii probiotic affect long-term establishment of the organism in the rumen and alter rumen metabolism in the dairy calf? J. Dairy Sci. 101:1747-1751. 10.3168/jds.2017-12551

Zhong, R. Z., H. X. Sun, G. D. Li, H. W. Liu, and D. W. Zhou. 2014. Effects of inoculation with rumen fluid on nutrient digestibility, growth performance and rumen fermentation of early weaned lambs. Livest. Sci. 162:154-158. 10.1016/j.livsci.2013.12.021

Ziolecka, A., Z. Osinska, and A. Ziolecki. 1984. The effect of Stabilized Rumen Extract on growth and development of calves. 1. Liveweight gain and efficiency of feed utilization. Z. Tierphysiol. Tierernahr. Futtermittelkd. 51:13-20.

Ziolecki, A., E. Kwiatkowska, and H. Laskowska. 1984. The effect of Stabilized Rumen Extract on growth and development of calves. 2. Digestive activity in the rumen and development of microflora in the rumen and faeces. Z. Tierphysiol. Tierernahr. Futtermittelkd. 51:20-31. 
Table 1. Time line for colonisation of the major bacteria phyla from birth to adulthood (values expressed as range of mean percentages)

\begin{tabular}{llllllll}
\hline & \multicolumn{7}{c}{ Age } \\
\cline { 2 - 7 } & 3-days & 7-days & 14-days & 28-days & 42-days & 6-months & 2-years \\
\hline Phyla & & & & & & & \\
Bacteroidetes & $13.9-42.6$ & $56.3-56.9$ & $46.0-61.3$ & $49.9-56.3$ & $56.3-74$ & $38.5-55.2$ & $38.5-50.2$ \\
Firmicutes & $5.1-13.9$ & $13.9-17.5$ & $13.9-34.0$ & $13.9-42.1$ & $10-43.9$ & $36.8-48.9$ & $34.5-56.7$ \\
Actinobacteria & $0.05-4.9$ & $0.6-4.9$ & $0.9-4.9$ & $0.3-4.9$ & $0.3-4.1$ & 3 & 3 \\
Fusobacteria & $4.7-5.5$ & $4.7-5.3$ & $0.2-0.6$ & $0.2-0.3$ & $0.2-0.4$ & 0.1 & 0.1 \\
Spirochaetes & $0-0.4$ & $0.1-0.4$ & $0.4-2.6$ & $0.4-0.9$ & 0.4 & $0.7-1.2$ & $0.9-2.5$ \\
Fibrobacteres & $0-0.3$ & $0-0.3$ & $0.2-0.3$ & $0.3-1.5$ & $0.3-1.6$ & $0.2-1.7$ & $0.5-2.1$ \\
Tenericutes & 0 & 0.8 & 0.2 & 0.9 & 1.0 & $1.0-1.6$ & $1.3-2.3$ \\
\hline Data collected from Li et al. (2012); Jamie et al. (2013); Rey et al. (2013); Yáñez-Ruzi et al. (2015); Abecia et al. (2018)
\end{tabular}




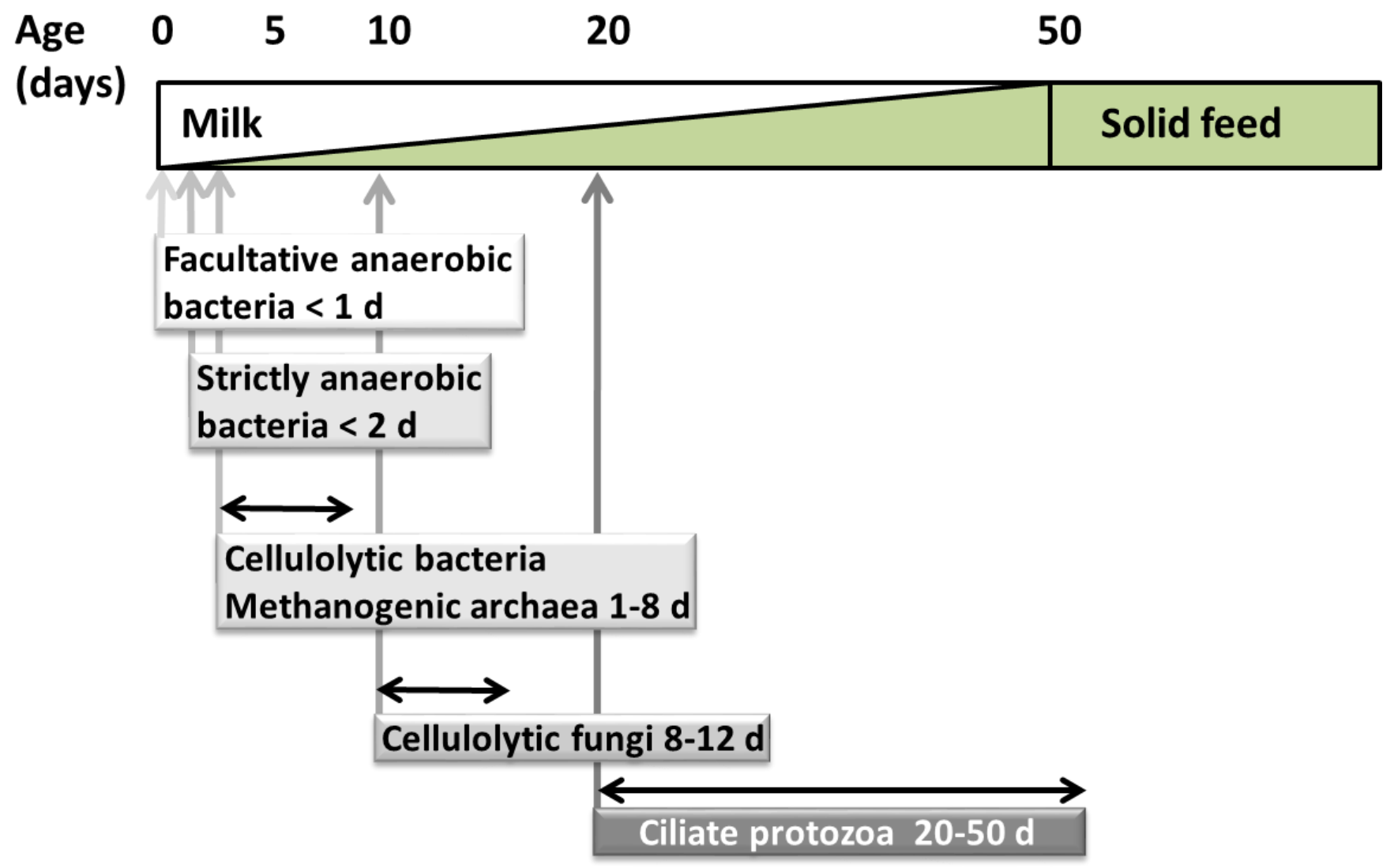

Figure 1. Microbial colonisation of the lamb rumen. Colonisation of the rumen by microbial groups detected by cultural and molecular methods. Lambs were kept with their dams. Arrows indicate the start of the colonization period. 


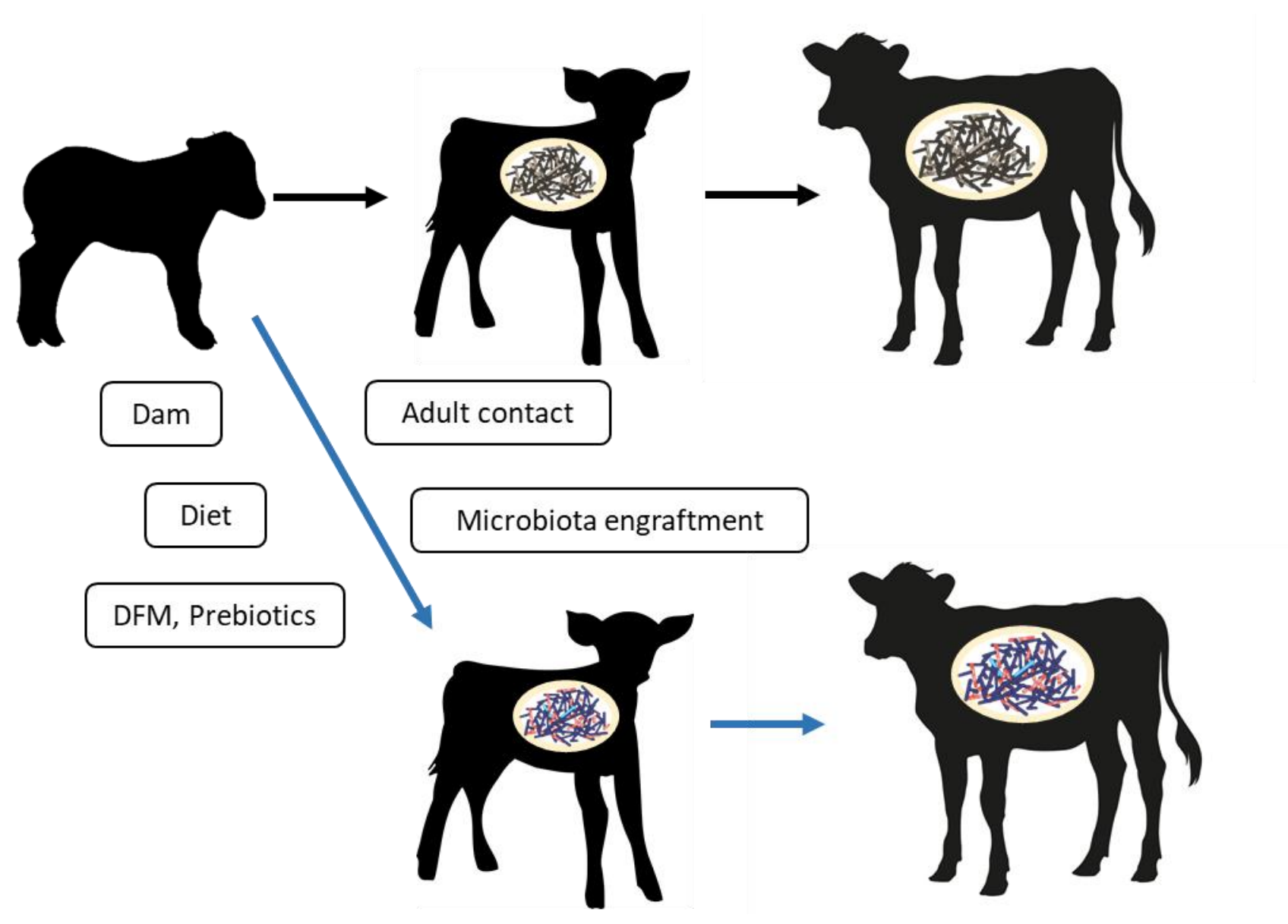

Figure 2. Acquisition of gastrointestinal microbiota in early life could be modulated (blue arrows) through management and targeted interventions to favour health and phenotypes of interest such as increased feed efficiency and decreased methane emissions.

Modulating options are: allowing contact with dams or mature conspecific harbouring a desirable microbiota, inoculation with gastrointestinal contents (engraftment) obtained from mature conspecific, diet and supplementation with direct-fed microbials (DFM), prebiotics and feed additives. 